\title{
How do energetic ions damage metallic surfaces?
}

Y.N. Osetsky*†, A.F. Calderł and R.E. Stoller $\uparrow$

$†$ Materials Science and Technology Division, ORNL,

Oak Ridge, TN 37831-6138, USA

\$Department of Engineering, University of Liverpool, Brownlow Hill, Liverpool, L69 3GH, UK

\begin{abstract}
Surface modification of structural and functional materials under bombardment by energetic ions is observed under different conditions in and can be either an unavoidable effect of the irradiation or an intentional modification to enhance materials properties. Understanding the basic mechanisms is necessary for predicting property changes. The mechanisms activated during ion irradiation are of atomic scale and atomic scale modeling is the most suitable tool to study these processes. In this paper, we present results of an extensive simulation program aimed at developing an understanding of primary surface damage in iron induced by energetic particles. We simulated $25 \mathrm{keV}$ self-ion bombardment of Fe thin films with (100) and (110) surfaces at room temperature. A large number of simulations, $\sim 400$, were carried out allow a statistically significant treatment of the results. The particular mechanism of surface damage depends on how the destructive supersonic shock wave generated by the displacement cascade interacts with the free surface. Three scenarios were primarily observed, with the limiting cases being damage created far below the surface with little or no impact on the surface itself, and extensive direct surface damage on the timescale of a few picoseconds. In some cases, formation of large $<100>$ vacancy loops beneath the free surface was observed, which may explain some earlier experimental observations.
\end{abstract}

Kevwords: radiation damage, displacement cascade, surface modification, shock wave, Iron

*Author for correspondence. Email: osetskiyyn@ornl.gov 


\section{Introduction}

Exposure to energetic particle irradiation can change the dimensions, and the mechanical and physical properties of materials. These effects are due to microstructural changes that evolve with time and arise from the production of radiation damage. The primary damage is produced in displacement cascades by the recoil of primary knock-on atoms (PKAs) and consists of point defects (vacancies and interstitials) and their clusters. Damage formation depends strongly on the type and energy of the irradiating particle. Neutron irradiation creates displacement cascades uniformly throughout a specimen (here called bulk cascades) while the damage from ion irradiation is restricted to a region near the surface where the ions enter. Irradiation by ions may be used to investigate radiation damage evolution similar to that produced by neutron irradiation (but without the inconvenience of induced radioactivity), or to modify material properties by impurity implantation, creation of sub-surface defects, and changing the surface structure. For typical radiation damage investigations using self-ions with energies of a few $\mathrm{MeV}$, the thickness of this damaged layer is on the order of a few micrometers for metals such as copper or iron [1]. Lower energy ions, a few tens of $\mathrm{keV}$, are typically used in fundamental studies involving in situ irradiation of thin foils ( $\sim 100 \mathrm{~nm}$ thick) in a transmission electron microscope [2]. Ions in this lower energy range are also used in the solid state processing practices of the electronics industry [3] and for the purpose of surface modification [4].In these latter cases the and the corresponding damage layer is a few tens of nanometers thick. The number of vacancies and interstitials produced per cascade, the fraction of them in clusters and the degree of surface modification vary with incident atom/ion energy and mass, the temperature, and the material itself.

In recent years, much research in the field of neutron-induced radiation damage has focused on interstitial clusters, in recognition of the fact that their thermal stability, onedimensional mobility and interaction with impurity atoms provide a strong driving force for damage accumulation and are key elements in explaining a number of experimental observations [5]. However, near-surface damage is influenced by the existence of the nearby free surface which may affect either the evolution of the displacement cascade itself or the short-term, postcascade defect evolution. Previous atomistic studies of ion surface irradiations focused on facecentered cubic (fcc) metals and a few specific mechanisms have been described [6-10]. For example, three types of surface damage mechanisms in fcc metals $\mathrm{Pt}, \mathrm{Au}, \mathrm{Cu}$ and $\mathrm{Ni}$ and in $\mathrm{Ge}$ are described in [7]. Type I was associated with what the authors called "linear cascades". These 
cascades do not form a thermal spike and evolve via atomic replacement sequences and occur for incident ions with low energy. They create minimal sub-surface damage and a limited amount of surface modification, creating only a few adatoms and small pits. Type II cascades were associated with a thermal spike and a corresponding local melting that produced viscous flow of material towards the free surface. These cascades produced a crater and rim of adatoms. Vacancy cluster formation was explained by cavitation in the melted region. Finally, the behavior of type III cascades was attributed to "microexplosions". They occur when the center of energy in a dense cascade lies very close to the surface and lead to the production of many sputtered atoms and a crater on the surface.

Predicting cascade evolution and the formation of surface damage under ion irradiation is a rather difficult problem that has been investigated for only a few particular cases. Some not very successful attempts have been made to estimate the number of adatoms created under type II cascade conditions on the basis of elastic and viscous properties of the target material and energy of incident ion [7]. A detailed study of surface crater formation and a prediction of their probability was made in [10] for the case of gold irradiated by Xe ions with energies up to $100 \mathrm{keV}$. It was concluded that the cratering probability increases at high energy. A phenomenological model for predicting the cratering probability was suggested as a further development of the model described in [7]. However, the physical background for this model is rather obscure. Other studies have focused on the mechanisms of sputtering. For example, results of irradiation by low (few hundred eV) and moderate energy (few keV) ions were discussed in [10-18]. Sputtering by atoms or ions with a few $\mathrm{keV}$ of kinetic energy was modeled in many materials and significant progress was achieved in understanding and development of predictive models $[19,20]$. Sputtering due to direct collisions (Type I) can be well predicted for low-energy incident ions using binary collision models; however, under thermal spike conditions the interactions become more complicated and involve more than kinetic energy redistribution.

Among other observations reported from surface radiation damage studies in fcc metals we mention the formation of complicated adatom structures on the irradiated surface [8] and long range one-dimensional glide of large interstitial dislocation loops towards the free surface [9]. The impact of the one-dimensional glide mechanism was later discussed for other fcc metals in more detail [14]. Limited information is available on modeling surface primary damage in metals with a body-centered cubic (bcc) lattice. For example, 10 and $20 \mathrm{keV}$ self-ion irradiation was 
simulated in $[21,22]$. In that work, two series of cascades simulations were carried out and compared to the results of simulations without a free surface. In one series, the cascades were initiated using a surface PKA, and in the other, an atom 10 lattice parameters $\left(\mathrm{a}_{\mathrm{o}}\right)$ below the surface. A clear effect of the surface on defect formation was observed, with results from cascades initiated $10 \mathrm{a}_{\mathrm{o}}$ below the surface more similar to bulk cascades than the cascades initiated at the surface. For example, equal numbers of vacancies and interstitials are always formed in bulk cascades and the same result was observed in the $10 \mathrm{a}_{\mathrm{o}}$ cascades. However, an excess of vacancies survived in the surface cascades due to sputtering and the loss of mobile interstitial defects to the free surface (creating adatoms). Increased formation of vacancy clusters and larger vacancy clusters were observed in the surface cascades. The results of fifty cascades initiated by $48.5 \mathrm{keV} \mathrm{Xe}$ atoms simulated in Mo over the wide temperature range of 250-2500K are discussed in [23]. Extensive formation of large $1 / 2<111>$ vacancy loops was observed and explained by a melting and fast recrystallization mechanism that is somewhat similar to the Type 3 cascades described above as "microexplosions".

Although sputtering has been well investigated [10-20], it is only one component of the damage induced by near-surface displacement cascades, and we conclude that the available information on ion irradiation damage in bec metals is not sufficient to provide a clear understanding of all the mechanisms involved in creating near-surface damage. Moreover, the picture drawn for fcc metals is clear only for the effect of incident ion energy. There is a reasonable level of understanding of ion radiation damage mechanisms in two extreme cases:

1) very high-energy ions, that produce the characteristic bulk damage mechanisms without any significant effect of the free surface on defect evolution at the later stages;

2) low-energy ions, that produce damage similar to that in what was called "linear" cascades in [7]. These individual events create quite subtle surface damage which can be described by energy partitioning during binary collisions with relatively high accuracy.

The most complicated case is when a thermal spike occurs near the surface. A limited investigation of such events has shown a variation of damage mechanisms that were by no means rationalized or well described.

Recently, an extended investigation of bulk cascades in Fe has clarified the mechanisms of high-energy cascade evolution [24, 25]. High energy here means energy close to the maximum before the extensive subcascade formation; for Fe self-ions this is about $25 \mathrm{keV}$. It was found 
that for Fe and heavier PKAs of about this energy the formation and propagation of a supersonic shock front defines cascade evolution and primary damage. It was demonstrated that extensive defect formation occurs in the supersonic shock front and its life time determines the number of defects that survive after it is dissipated. The longer the supersonic phase lasts, the more separated are the vacancy and interstitial components of the primary damage and more defects survive. This is associated with more compact cascades with a higher local energy density. Special attention was paid to the effect of supersonic front propagation through locally nonhomogenous media, such as a region where a smaller subcascade has been initiated. It was systematically observed that such cases result in larger defect clusters of both vacancy and interstitial type (see schematic description in Fig. 11 of [24]). The explanation of material flow in the front of cascade-induced shock wave towards a neighboring subcascade region is quite similar, at least qualitatively, to the explanation of the Types 2 and 3 cascades discussed above, i.e. it is appears similar to plastic flow and "microexplosions" (see discussion and Fig. 13 in [7]). The variation in cascade behavior observed near surfaces is much higher than that in bulk and, unlike bulk cascades, there are no simple descriptors suitable for even a qualitative estimate of their average behavior. For example, simulation of bulk cascades has revealed a simple expression for the number of Frenkel pairs, $N_{F}$, created for a given cascade energy, $E_{C}: N_{F}=$ $A\left(E_{C}\right)^{m}$ [26]. This was confirmed in many independent simulations and the parameters $A$ and $m$ were found to be slightly material and temperature dependent, with different values in different energy regimes $[26,28]$. The differences are a result of the fact that basic mechanisms of cascade evolution are different for heavy and light materials. Nevertheless, a basic understanding of bulk cascade development, including the role of the thermal spike and shock wave in high-energy cascades, mechanisms of vacancy and interstitial cluster formation and recombination, and the statistics of defect cluster formation was obtained for many materials. A comparison with the standard NRT secondary displacement model for estimating defect production [29,30] has demonstrated that the number of stable defects produced in molecular dynamics (MD) simulation of bulk cascades is about $10-35 \%$ of NRT value for PKA energies relevant to neutron irradiation [31]. The situation for the near-surface cascades is much more complicated because of the large variety of mechanisms that can be involved and the absence of practical models for their general description. So far, primarily sputtering by relatively low energy ions, below a few keV, has been more or less successfully described [18-20]. 
In this paper we describe the results of our research to characterize the detailed evolution of near-surface cascade damage production and near-surface damage mechanisms when thermal spike conditions are realized. The primary message is that the particular damage mechanisms observed depend sensitively on how the supersonic shock front generated by the energetic ion interacts with the free surface. Details of the MD simulations are summarized in section 2. The results are presented in section 3 with the main focus on the degree of similarity between shock wave propagation in bulk and near-surface cascades. Classification of different types of cascades from the point of view of the supersonic shock front interaction with the free surface is presented in section 4 together with some characteristic examples of surface damage and modification. The results are discussed in section 5 and the conclusions are drawn in section 6 .

\section{MD simulations}

Because of the absence of any reliable scheme for quantitative characterization of nearsurface damage, we will first present a qualitative description of the results and then characterize them from the point of view the development of the destructive shock wave and its interaction with the free surface. We should underline here that this approach is valid for the following specific ion irradiation conditions:

1. Incident ion energy is low enough and its mass is high enough to prevent deep penetration into the interior of the sample.

2. Incident ion energy and mass are high enough to create cascades that are sufficiently dense to generate destructive supersonic shock waves.

Therefore, intentionally we avoid cases related to both low-energy ion surface implantation and so-called swift heavy ions for which some descriptive models are already exist.

The MD model of Fe was the same as that described in $[24,25]$ with the exception that free surfaces were used on to parallel faces to simulate a thin film with "top" and "bottom" surfaces; conventional periodic boundary conditions were used in the two orthogonal directions. The simulated crystal contained between 2 and 9.5 million atoms depending on the thin film thickness, which was 34 to $57 \mathrm{~nm}$, respectively. Atomic interactions were computed using the embedded-atom-method empirical potential from [32]. The short-range $(\leq 0.1 \mathrm{~nm})$ pair part of the potential was replaced by the Universal screened-Coulomb potential [33] in order to accurately describe the high-energy atomic collisions that occur in cascades. All simulations were done at 
$300 \mathrm{~K}$. Special attention was paid to thermal equilibration of the thin film to damp vibration of the free surfaces prior to the injection of the ion. Full equilibration required 3-5 stochastic redistributions of atomic velocity distribution and annealing over $\sim 100 \mathrm{ps}$. After free surfaces were quiescent, the cascade event was initiated by giving an $\mathrm{Fe}$ atom a velocity corresponding to $25 \mathrm{keV}\left(\mathrm{v}=2 E_{\mathrm{C}} / \mathrm{PFe}\right)^{1 / 2}$ directed towards the free surface from a distance of $\sim 2$ lattice parameters which is approximately the range of the interatomic potential employed. The direction of the incident $\mathrm{Fe}$ atom was $\sim 30^{\circ}$ from the incident surface normal to minimize channelling effects. Multiple cascades were simulated by changing the initial position of the incident atom, its particular velocity direction vector, and the time of the event initiation in order to obtain a statistically significant description of cascade evolution. We have simulated $\sim 100$ events in different crystals of 2 and 9.5 million atoms and 150 events in each of two 4.5 million atoms crystals with (100) and (110) incident surfaces.

In the case of bulk cascades, defect production is typically accomplished using an analysis based on either Wigner-Seitz cells or a characterization of displaced atoms [25]. These allow the total number of vacancies and interstitials to be counted and the number and size distribution of point defect clusters to be determined. Although equal numbers of vacancies and interstitials are always produced in bulk cascades, this is not the case for near-surface cascades. In addition several other types of defects and defect structures are produced in the latter case. These include: sputtered atoms, adatoms on the free surfaces and surface topology changes such as regular elevations/depressions, pits or craters. The variety of defects, together with the imbalance of vacancies and interstitials, makes identification, cataloging and a statistical description of damage from near-cascade damage much more complicated. To analyze the cascades, we have used both the Wigner-Seitz cell and displaced atom approaches together with the chain-ofdisplacements or "spaghetti" analysis from [25] and the surface triangulation analysis within the PyMol software [25, 34]. The cascade 'spaghetti' introduced in [25] is an analysis of the chain of site-to-site atomic displacements (not individual atom trajectories) that lead to interstitial formation. In a cascade involving permanent displacement of hundreds or thousands of atoms, nearly all site-to-site lines form a chain of connectors between sites in a sequence of atomic replacements, and there may be many such chains in the cascade zone. In bulk cascades, a vacancy resides at the beginning of the chain and the ultimate interstitial is at the end. This analysis is very useful in elucidating the mechanisms involved in stable defect production. In the 
context of the present paper, when a general picture of cascade evolution is needed, we use the total number of vacancies which is the number of Wigner-Seitz cells with no atom inside.

\section{Displacement cascade dynamics}

The terminology shock front implies an abrupt change in pressure, density and temperature (atomic velocity). In [25] the correspondence between the dynamics of defect production and shock wave propagation was unambiguously established. To demonstrate the similarities between the cases of bulk and surface irradiation, the evolution of the number of empty WignerSeitz cells versus time is shown in Figs. 1. Fig. 1a shows results of 300 cascades modeled in crystals with $\sim 4.5$ million atoms and (100) and (110) incident surfaces. To provide a better view of the evolution, Fig. 1b includes only the maximum and the minimum cases for both of the surface orientations. This does not reveal the detailed complexity of the dynamics of defect formation during cascade evolution because only the total number of vacancy-type defects is shown. However, a characteristic change of the slope is observed for the vast majority of the presented events at about 0.1 to $0.2 \mathrm{ps}$. In [25] this change in slope was attributed to the change in velocity of the shock front from the destructive supersonic to a non-destructive subsonic phase in which the associated deformation is purely elastic.

As the comparison of Figs. $1 \mathrm{a}$ and $1 \mathrm{~b}$ indicates, the damage evolution is similar for all the cascades, including "bulk" and "near-surface" ones. First, up to $t \sim 0.1 \mathrm{ps,} \mathrm{the} \mathrm{number} \mathrm{of} \mathrm{empty}$ Wigner-Seitz cells is quite linear vs time (note the log-log scale in Figs. 1). That means the number of defects is proportional to $t^{n}$ with the exponent $n$ being approximately constant for a particular cascade and varying between 2.5 and 3 for different cascades. This implies a damage zone front moving with approximately constant velocity and creating new defects at a nearly constant rate at this stage. Analysis reported in [25] revealed that the front moves outwards with a speed of approximately $30 \mathrm{~km} \mathrm{~s}^{-1}$, i.e. about five times the longitudinal velocity of sound, $v_{\mathrm{s}}$, in iron. The front velocity is supersonic in character up to $t \sim 0.1 \mathrm{ps}$. Note that in some very dense cascades initiated by heavy or molecular ions the shock front may reach a velocity up to $10 v_{\mathrm{s}}$. Second, after $\sim 0.1 \mathrm{ps}$, the production rate of new defects slows to become approximately proportional to $t$ because the shock front velocity decreases to sonic or lower speed as $t$ approaches $\sim 1$ ps. Finally, the shock front dissipates after $\sim 1$ ps and no additional defects are 
produced. Beyond this point, the number of vacancies and interstitials are rapidly reduced by recombination.

It should be pointed out that the empty Wigner-Seitz cells observed at short times do not necessarily correspond to stable lattice vacancies in the normal sense. The passage of the shock front moves many atoms just enough to remove the atom from the cell, and much of what is called in-cascade recombination is simply the relaxation of these atoms back into the same or an adjacent cell. In this way, both point defect "formation" and "recombination" occur simultaneously during the time the number of empty Wigner-Seitz cells is increasing. Even during the time when the net number of empty Wigner-Seitz cells is initially decreasing, there may be some creation of empty cells and apparent interstitials. Differences in the recombinationdominated phase give rise to the quite different behavior observed for the extreme cases presented in Fig. 1b. Cascades with a low number of surviving defects demonstrate very intensive recombination initially with a dependence close to that of $t^{-1}$ which may accelerate to $t^{-}$ 2 . For such cases the number of stable defects is within the range of results observed for bulk cascades $[25,26]$ and indeed such cascades developed well below the free surface and no interaction or defect exchange was observed with either free surface. We therefore also designate these cascades as "bulk".

The other extreme in Fig. 1b demonstrates more limited recombination at a lower rate. These cascades produced substantial damage near the surface that included a few tens of sputtered atoms, more than a thousand ad-atoms and craters with a depth of up to 15 lattice parameters containing more than 1400 vacancies. It is interesting that the final number of empty Wigner-Seitz cells is very close to the corresponding number at the time when the supersonic shock front slows down to sonic speeds, i.e. $\sim 0.1$ ps. In these cases it appears that the defects formed after this transonic transformation were removed during the recombination phase. Features and defects such as deep craters, adatoms and sputtered atoms are practically all formed by the time of transonic transformation. It is the primarily the defects produced well below the surface that recombine as in bulk cascades while the surface damage remains following cascade annealing. It is also noticeable in Fig. 1b that two of the cascades developed more rapidly than the other two cascades. This is because for the latter two the incident atom penetrated more deeply into the bulk before the intensive collisions started. These cascades with the maximum number of retained defects in Fig. 1b represent the extreme case of what we refer to here as 
"surface" cascades; they are created when the supersonic shock front intersects the free surface shortly after its formation. Formation and propagation of the shock wave for such a case is demonstrated in Fig. 2, showing the distribution of local atomic density during different stages for a (100) surface cascade. Each atom in the (110) cross-section is indicated with a symbol whose color depends on the number of atoms within the second-nearest-neighbor distance of $\sim 1.4$ lattice parameters; the darker color, the more atoms are found. The darkest symbols, which correspond to more than 26 atoms (compared to 20 for the perfect lattice), clearly show propagation of the high-density region which is the shock wave front. A full movie of the shock front propagation is attached as supplementary material.

The other cases of defect evolution presented in Fig. 1a are distributed between these two extremes, i.e. between bulk cascades and surface cascades. Qualitative analysis of the final damage allows one to define a third characteristic example, the "near-surface" cascade, which develops when the transonic shock front transformation occurs just below the surface. All the qualitative results are presented and described in the next section.

\section{Types of ion-induced cascades from the point of view shock wave propagation.}

An illustration of the transonic shock front and a corresponding example of an ionproduced bulk cascade are presented in Fig. 3. Fig. 3a illustrates the case in which the shock front becomes subsonic, and there is no additional defect production, before it reaches the free surface and the core cascade damage is produced well below the surface. A typical example of this type of cascade is shown in Fig. $3 \mathrm{~b}$ with the atomic replacements highlighted by a "spaghetti" diagram. The cascade evolution presented in Fig. 3b for an ion-produced bulk cascade is similar to that observed earlier $[25,26]$ when simulating bulk cascades from neutron irradiation. In this example, the entire cascade volume, represented by the "spaghetti" diagram which visualizes chains of atomic replacements, does not significantly interact with the free surface. The number of defects generated here are nearly balanced between vacancies (50) and interstitials (49) in the bulk, with 3 surface vacancies, and 4 adatoms. There are no large clusters which is similar to the earlier simulations of bulk cascades $[25,26]$ with a self-PKA.

The case of a "near-surface" cascade presented in Fig. 4 was observed when the transonic transition of the shock front occurred very near or slightly overlapped the free surface. The destructive supersonic shock front interacting with the free surface still had enough energy to 
push atoms onto the surface, creating a mound of adatoms. There is no significant sputtering because the kinetic energy of the adatoms was not high enough to escape the surface. There is a vacancy cluster below the surface that contains roughly the same number of vacancies as the number of adatoms. The example presented in Fig. 4b is for a (001) surface cascade and contains the following defects: sputtered atoms (10); adatoms (645); bulk interstitials (33), including a 15interstitial cluster; and bulk vacancies (687) including a 650-vacancy loop with the Burger vector $<010>$ and an irregular cluster of 30 vacancies attached to the loop.

A second example of a "near-surface" event is shown in Fig. 5. This involved large vacancy loops created with a $1 / 2<111>$ Burgers vector with a glide plane that intersected the free surface. The loops glided to the surface creating loop-shaped depressions with a depth equal to the loop Burgers vector. These surface features created by vacancy loops are reported here for the first time. It is interesting that glide of vacancy loops formed in near-surface cascades and the formation of these regular depressions has not been reported previously since the formation of elevated regions due the to glide of $1 / 2<110>$ interstitial loops to the free surface in fcc metals has been reported [7]. Similar elevated structures formed by gliding $1 / 2<111>$ interstitial loops were observed in the present work and an example is presented in Fig. 5c. . When an interstitial loop reaches the surface, it creates a loop-shaped region of elevation with a height of its Burgers vector. . Geological features called "grabens" and "horsts" are regions depressed or elevated relative to the surrounding terrain due to horizontal tension or compression in the earth crust as shown in Ref. [35]. Geometrically they look very similar to the structures observed here due to the glide of large vacancy or interstitial loops to the free surface. The case presented in Fig. 5b contains 22 sputtered atoms, a 354 adatom mound, 11 bulk interstitials, 2 "grabens" formed due to the glide of 148 and 180 vacancy loops with Burgers vectors of $1 / 2<111>$ type intersecting the (001) surface and 44 bulk vacancies. The horst-like structures on the surface in Fig. 5c were observed for the first time in modeling $50 \mathrm{keV}$ surface cascades in $\mathrm{Cu}$ [9]. The most significant damage occurs when the destructive supersonic shock front intersects the free surface as shown in Fig. 6. Many atoms which have high kinetic energy are sputtered while others create a rim of adatoms around a huge crater. In some cases there are vacancy loops below the surface that always have a $<100>$-type Burgers vector. This is the case presented in Figs. 6(b-d) where the damage consists of: interstitials (6), vacancies (215), crater (1345 vacancies), adatoms (1440) and sputtered atoms (98). 
An example of the same cascade type, but with the vacancy loops having a Burgers vector intersecting with the free surface is presented schematically in Fig. 7a. For the particular case presented in Fig. $7 \mathrm{~b}$ the damage includes 21 sputtered atoms, 338 adatoms in the rim of 159 vacancy crater, a [010] loop of 89 vacancies, a "graben" created on the surface due to glide of a $1 / 2<111>$ loop of 115 vacancies, and 16 interstitial in the bulk. Note, that for typical "surface" cascades we have observed the formation of vacancy loops and "grabens," but not large interstitial loops or "horsts."

\section{Discussion}

About 400 surface cascades with a PKA energy of $25 \mathrm{keV}$ were simulated with the aim of observing and characterizing the wide range of different defect formation mechanisms which occur under conditions which involve the formation of a supersonic shock wave. Although such conditions are relevant to radiation damage experiments which employ ion irradiation in situ for observation by transmission electron microscopy, according to the literature, they are the least investigated by computational modeling. Some of the mechanisms observed in this study have described previously, while others were observed for the first time. Among the new mechanisms was the generation of glissile vacancy loops with a $1 / 2<111>$ Burgers vector and their glide to the free surface, leading to the formation of depressed areas with a depth equal to the Burgers vector. The depressed areas are similar to the geological feature known as a "graben". The variety of observed mechanisms is too broad to permit us to extract a single (or even a few) parameter(s) which could be used to predict specific characteristics of defect formation such as the total number of stable defects or the degree of defect cluster formation. However, we identified a descriptor that allowed us make a qualitative separation of the mechanisms. This is the position of the center of mass of the displacement cascade relative to the free surface which determines the interaction of the destructive supersonic shock wave front with the surface. The characteristic properties of the three main mechanisms are described in Figs. 3, 4 and 6. Note that the damage produced by the two last mechanisms, namely "near-surface" and "surface" cascades, is similar to that referred to in [7] as the "thermal spike" and "microexplosion" mechanisms (see Figs. 13 b and $\mathrm{c}$ in [7]). The cascade behavior in most of our simulations lies between the extremes illustrated in Fig. 1b because the transonic transformation occurs at different distances from the free surface. The spectrum of these distances is practically continuous, leading to the smooth 
transformation from one mechanisms into the another. In the present research we have attempted to carry out enough simulations to obtain some statistically significant information about the mechanisms. Fig. 1a demonstrates that the final number of defects produced is distributed more or less uniformly between the extreme cases (indicated in Fig. 1a). This dispersion makes development of an analytical descriptive or/and predictive model very difficult if not impossible. A particular model may describe a particular mechanism, for example as it is the case of lowenergy ion sputtering. It is extremely difficult to develop a multi-mechanism model for the cases observed here.

The importance of supersonic shock front propagation on defect production was described in detail in [25]. It was demonstrated that the largest clusters of both vacancy and interstitial type are formed when the supersonic shock front intersects a density fluctuation in the crystal such as a nearby subcascade (Fig. 11 in Ref. [25]). This is fully consistent with the behavior of surface cascades observed here; the free surface can also serve as such a fluctuation and the mechanisms discussed in [25] can be generalized for the case of surface cascades. Indeed, in the current research this mechanism also produces the maximum possible damage. Rather than the large interstitial clusters observed in bulk cascades, the surface cascades produce numerous adatoms and sputtered atoms, and very large vacancy loops and surface craters are created rather that the vacancy clusters observed in bulk cascades. It is not surprising that the effect of the free surface is much stronger than that of a nearby subcascade because it represents a much larger density fluctuation. The total damage due to surface cascades can be about an order of magnitude larger than in the case bulk cascades. The dynamics of bulk and surface cascades is shown in Figs. 1 and the largest difference is in the annealing stage. The number of defects in bulk cascades decreases $\sim 100$ times during this stage due to extensive vacancy-interstitial recombination. On the contrary, the destructive supersonic shock front produces irreversible damage in surface cascades, expelling hundreds of atoms to the surface and farther and the effect of under-surface defect recombination decreases significantly. It is reasonable to conclude that the behavior of surface cascades observed here as well as "microexplosions" observed in $[4,10,23]$ are just the extreme case of the mechanism discussed in [25].

The depth below the surface at which the first high-angle collision is observed largely determines which of the three types of cascade evolution is followed. Since the SRIM code [36] is widely used to predict damage phenomena under ion irradiation conditions, it is interesting to 
compare the results ion transport in our MD simulations with the predictions of SRIM. Using SRIM, we simulated self-ion irradiation in Fe with the same incident angle $30^{\circ}$ as in the MD simulations. Note that the SRIM code includes electronic stopping effects which were not accounted for in the MD simulations. Using the approximation of Ref. [29] to account for electronic stopping, the $25 \mathrm{keV}$ incident ion energy in MD simulations is equivalent to a PKA energy of $36.7 \mathrm{keV}$ which was therefore used in the SRIM calculations. The final depth of the incident ion from 1000 SRIM cascades is compared with the MD results for 150 events with both the (100) and (110) surfaces in Fig. 8. The SRIM results predict a slightly deeper average penetration depth of $12.8 \mathrm{~nm}$ compared with the MD values of $9.1 \mathrm{~nm}$ for (100) and $10.2 \mathrm{~nm}$ for (110) surfaces at $100 \mathrm{~K}$. Because of the effect of atomic vibrations on channeling, we expect that the MD results would be temperature dependent with the PKA penetration depth larger at low temperatures and smaller at high temperatures. This affect would not be reproduced in the amorphous target material in SRIM. Nevertheless, the qualitative results are surprisingly similar and the shape of all the distributions is practically the same. Although the ion transport cannot be directly related to the location of high-angle collisions and the thermal spike, SRIM results may be useful to develop a qualitative prediction of the damage under surface irradiation. Depthdependent information on ion-atom collisions can be obtained if SRIM is run in the "full cascade" mode.

Among all the MD simulations treated here, the main three mechanisms occur with roughly equal probability. We think that this is just a coincidence due to a combination of incident atom energy and temperature. Moreover, the specification of the three mechanisms reported is rather conditional for they are not fully distinct from one another and the spectrum of possible mechanisms is practically continuous as indicated by the results in Fig. 1. This makes it quite difficult to predict the results of ion irradiations. Nevertheless, some qualitative predictions are possible. Using SRIM to predict ion transport for different ion masses and energies, one could obtain both the location of collisions involving large energy transfer and the final depth of the incident ion (as in Fig. 8). Such information could be used to qualitatively predict the relative contribution from the different damage mechanisms. The larger the mean depths and high-angle collision locations, the deeper will be the cascade center and the transonic transformation will occur farther from the free surface. This will reduce the probability of the "surface" cascade mechanism. Of course, this is valid only for the cases in which the basic damage mechanism via 
a destructive supersonic shock wave is dominant due to the mass and energy of the incident ion. In the case of low energy ions the "linear cascade" mechanism described in [7] is more probable, while in the case of high-energy light atoms the bulk cascade behavior should dominate. Increasing incident ion mass decreases the penetration depth and increases the shock front power, increasing therefore the probability of "surface" cascades. Deviation of the incident ion direction from the surface normal also increases the probability of "surface" cascades and this effect can also be predicted qualitatively by combining SRIM simulations of the incident ion penetration probability with the current analysis of cascade mechanisms.

\section{Conclusions}

A large-scale program of molecular dynamics simulations involving $\sim 400$ collision cascades with $25 \mathrm{keV}$ Fe PKA incident on (100) and (110) Fe crystal surfaces was performed and the advanced visualization techniques used here have demonstrated both the shock front dynamics and how its interaction with the free surface determines the final damage. The primary observations from this study are:

1. Bulk cascades created deeply enough that the transformation from a supersonic to sonic shock front speed (transonic transformation) occurs far from the free surface do not directly produce surface damage.

2. Near-surface cascades in which this transonic transformation occurs close to the surface produce mounds of adatoms, sputtered atoms and large $<100>$ vacancy loops.

3. Surface cascades in which the supersonic front-shock interacts directly with the free surface produce deep craters with a rim of adatoms and large $<100>$ vacancy loops.

4. No large interstitial clusters were observed in any of the cascade simulations.

5. A new mechanism was observed of large vacancy loops gliding along $1 / 2<111>$ directions to the free surface and forming an extended regular depression similar to a geological "graben".

6. Ion irradiation is characterized by a very wide distribution of defect types and numbers of defects formed. For the same cascade energy of $25 \mathrm{keV}$, the number of stable defects ranged from $\sim 30$ Frenkel pair in bulk cascades to $>3500$ defects (vacancies, interstitials, adatoms and sputtered atoms) in surface cascades in the present simulations. 
7. The incident atom penetration depth depends slightly on the free surface crystallography, $9.1 \mathrm{~nm}$ and $10.2 \mathrm{~nm}$ for (100) and (110) surfaces, respectively. These values are smaller than the $12.8 \mathrm{~nm}$ obtained from SRIM simulations with the same parameters.

8. A method for making qualitative predictions of the relative importance of different damage mechanisms is suggested on the basis of SRIM simulations.

\section{Acknowledgments}

Research at the Oak Ridge National Laboratory sponsored by the U.S. Department of Energy, Office of Basic Energy Sciences, Materials Sciences and Engineering Division, "Center for Defect Physics," an Energy Frontier Research Center. 


\section{References:}

1. Farrell, K., Lewis, M. B., and Packan, N. H., Scripta Met., 1978: 12, 1121.

2. English, C.A. and Jenkins, M. L, Materials Science Forum 1987: 15-18, 1003

3. Tasch, A. F., Nucl. Inst. and Meth. in Physics Research B, 1993: 74, 3.

4. Ion Mixing and Surface Layer Alloying, Nicolet, M-A. and Picraux, S. T., Eds., Noyes Publications, Park Ridge, NJ, 1984.

5. Singh, B.N., Eldrup, M., and Horsewell, Ehrhart P. and F. Dworschak F., Philos. Mag., 2000: A 80, 2629.

6. Rehn, L.E., Averback, R.S. and Okamoto, P.R., Mater. Sci. and Eng., 1985: 69, 1.

7. Ghaly, M., Nordlund, K. and Averback, R., Philos. Mag., 1999: 4, 795.

8. Nordlund, K., Tarus, J., Keinonen, J., Donnelly, S.E. and Birtcher, R.C., Nucl. Instr. and Meth. in Phys. Res., 2003: B206, 189.

9. Nordlund K., Keinonen J., Ghaly M. and Averback R.S., Nature, 1999: 398, 48.

10. Bringa E.M., Nordlund K. and Keinonen J., Phys. Rev. 2001: 64, 236426.

11. Smith R., Kenny S.D. and Ramasawmy D., Phil. Trans. R. Soc., London, 2004: A362, 157-176.

12. Scott C. and Smith R., Proc. Roy. Soc. A Math. Phys. and Eng. Sci., 2013: 469, 20120480 .

13. Grigor'ev P. Yu. and Zhurkin E.E., J. Surf. Invest.: X-Ray, Synchrotron Neutron Tech, 2012: 6, 229.

14. Sang-Pil K., Huck Beng C., Eric C., Vivek B. S. and Kyung-Suk K., Proc. Roy. Soc. A Math. Phys. and Eng. Sci., 2012, 468, doi: 10.1098/rspa.2012.0042.

15. Li A. and Watts R.O., Journal of Chem. Phys.1995: 10,103(16).

16. Zhou X.W., Wadley H.N.G. and Sainathan S., Nucl. Instr. and Meth. in Phys. Res., 2005: B234, 441.

17. Zhurkin E.E., J. Surf. Invest.: X-Ray, Synchrotron Neutron Tech., 2009: 3, 192.

18. Sputtering by Particle Bombardment: Experiments and Computer Calculations from Threshold to MeV Energies, Ed. by R. Behrisch and W. Eckstein. Springer, 2007. ISSN electronic edition: $1437-0859$.

19. Weidtmann B., Duvenbeck A. and Wucher A., Appl. Surf. Sci., 2008: 255, 813. 
20. Kalyanasundaram N., Ghazisaeidi F., Freund J.B. and Johnson H.T., Appl. Phys. Lett., 2008: 92, 131909.

5. Stoller R.E., J.Nucl.Mater., 2002: 307-301, 935-940.

6. Stoller R.E. and S. G. Guiriec, J. Nucl. Mater., 2004: 329-333, 1228.

7. Starikov S.V., Insepov Z. and Rest J., Phys. Rev. 2011: B84, 104109.

8. Calder A., Barashev A.V., Bacon D.J and Osetsky Y.N, Philos. Mag. Lett., 2008: 88, 4353.

9. Calder A., Barashev A.V., Bacon D.J and Osetsky Y.N, Philos. Mag., 2010: 90, 863-884.

10. Bacon D.J., Calder A.F., Gao F., Kapinos V.G. and Wooding S.J., Nucl. Instrum. and Meth. 1995: B 102, 37.

11. Bacon D.J., Gao F. and Osetsky Y.N., J. Nucl. Mater, 2000: 276, 1-12.

12. Stoller R.E., J. of Nucl. Mater., 2000: 276, 22-32.

13. Norgett M.J., Robinson M.T. and Torrens I.M., Nucl. Eng. Design, 1975: 33, 50.

14. Standard E521, ASTM Annual Book of Standards, 1989.

15. Stoller R.E. and Greenwood L.R., J. of Nucl. Mater., 1999: 271 \& 272, 57.

16. Ackland G.J., Mendelev M.I., Srolovitz D.J., et al., J. Phys.: Condens. Matter., 2004: 16, S2629.

17. Biersack J.P. and Ziegler J.F., Nucl. Instrum. and Meth., 1982: 194, 93.

18. W.L. De Lano, The PyMOL Molecular Graphics System, DeLano Scientific LLC, Palo Alto, CA, USA (2008). http://www.pymol.org.

19. http://en.wikipedia.org/wiki/Graben

20. SRIM - The Stopping and Range of Ions in Matter, http://www.srim.org/ 


\section{Figure captions}

Fig. 1. Variation of the number of empty Wigner-Seitz cells versus time for (001) and (110) cascades: a) All 300 cascades and b) cascades producing the maximum and minimum number defects for each case.

Fig. 2. Snapshot cross-sections through the (001) "surface" cascade at various times. The cross-section plane is (01 $\overline{1})$ and the direction of the abscissa and ordinate is [100] and [011], respectively. The scale unit of length is the iron lattice parameter, $a_{0}$. The color (grey shading in the printed version of the paper) indicates the atomic neighbour density (the number of atoms in a sphere of radius $r_{\mathrm{c}}=1.405 a_{0}$ ) centered on the atom in question.

Fig. 3. Example of a bulk cascade: a) schematic representation of cascade development over time and typical "spaghetti" visualization, and b) large scale "spaghetti" diagram for a typical cascade.

Fig. 4. Example of a "near surface" cascade: a) schematic representation of cascade development over time and typical "spaghetti" visualization, and b) large scale "spaghetti" diagram for a typical cascade.

Fig. 5. Variant of a "near surface" cascade: a) schematic representation of cascade development over time and typical "spaghetti" visualization, b) large scale "spaghetti" diagram for a cascade in which two glissile vacancy loops formed and glided to the surface, and c) large scale "spaghetti" diagram for a cascade in which an interstitial loop formed and glided to the surface.

Fig. 6. Example of a "surface" cascade: a) schematic representation of cascade development over time and typical "spaghetti" visualization, and b-d) Large scale "spaghetti" diagram and closer view of the crater and vacancy loop.

Fig. 7. Variant of the "surface" cascade: a) schematic representation of cascade development over time and typical "spaghetti" visualization, and b) large scale "spaghetti" diagram for a "surface" cascade in which a deep crater, adatom mound and two vacancy loops formed and of them glided to the surface.

Fig. 8 Distribution of the final depths of incident ions obtained from 150 MD simulations of $25 \mathrm{keV}$ cascades for both (001) and (110) surfaces, and from a SRIM simulation of 1000 cascades with an ion energy of $36.7 \mathrm{keV}$ (see text for explanation of SRIM energy). 
Figures.

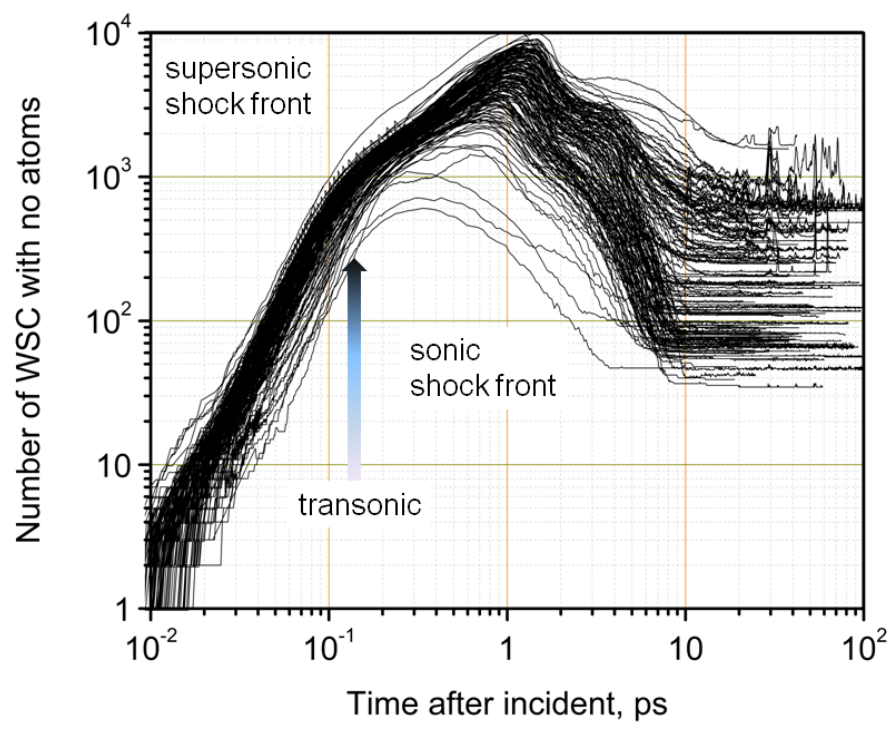

Fig.1a

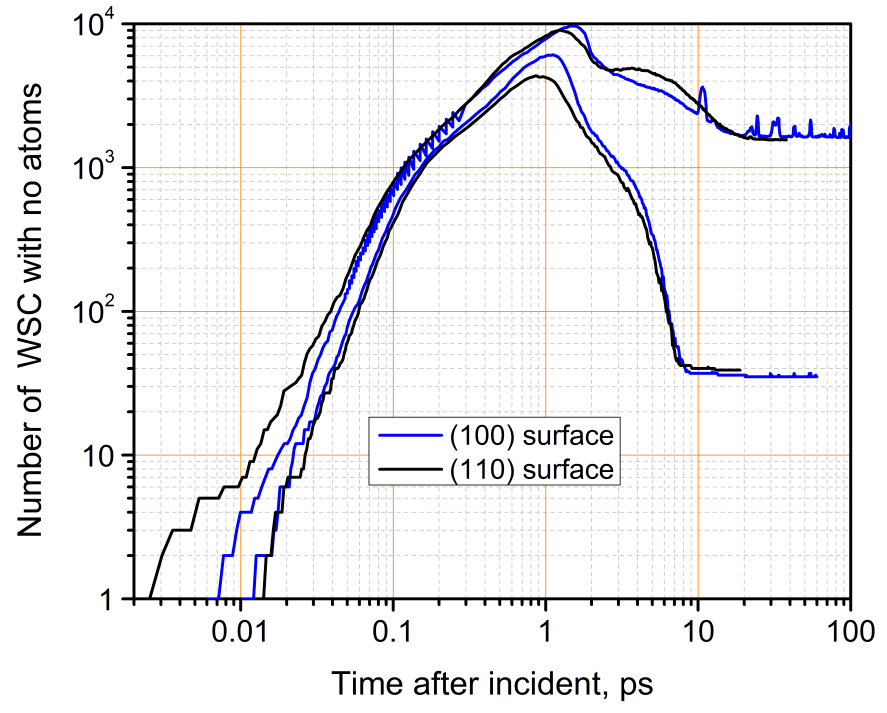

Fig.1b 


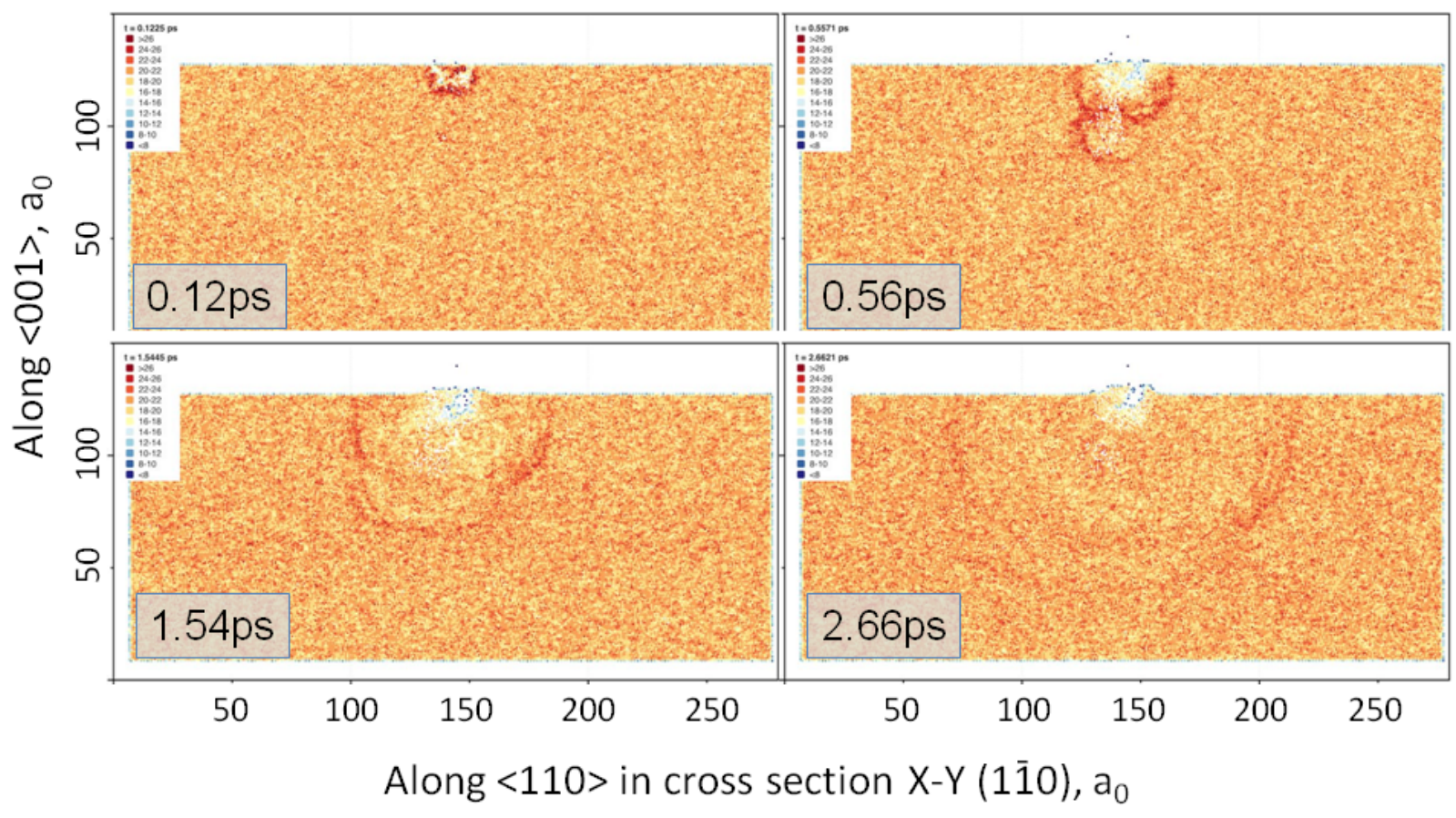

Fig.2. 


\begin{tabular}{|c|c|c|c|c|}
\hline doep & Free surface & few final defects \& little surface damage \\
\hline supersonic & shock & Transonic \\
boundary
\end{tabular}

Fig. 3a

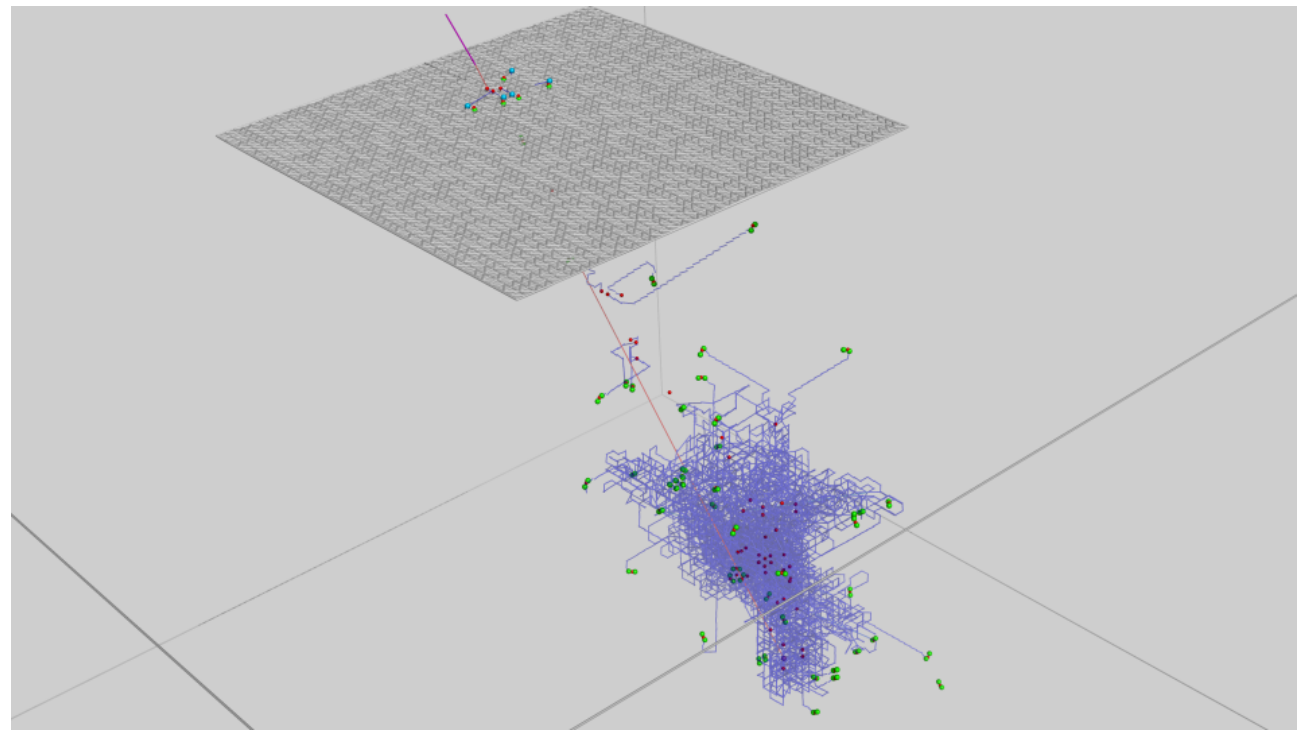

Fig.3b. 


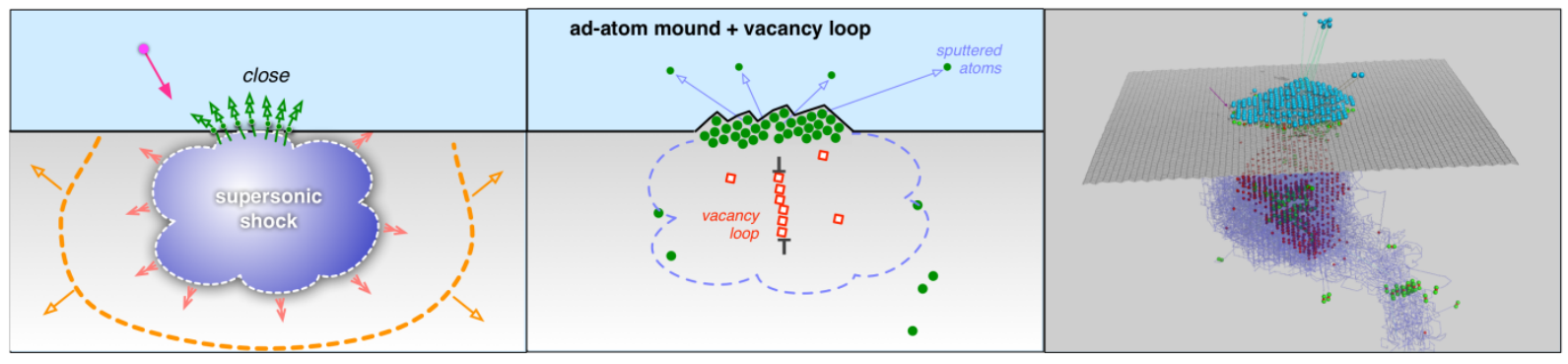

Fig.4a

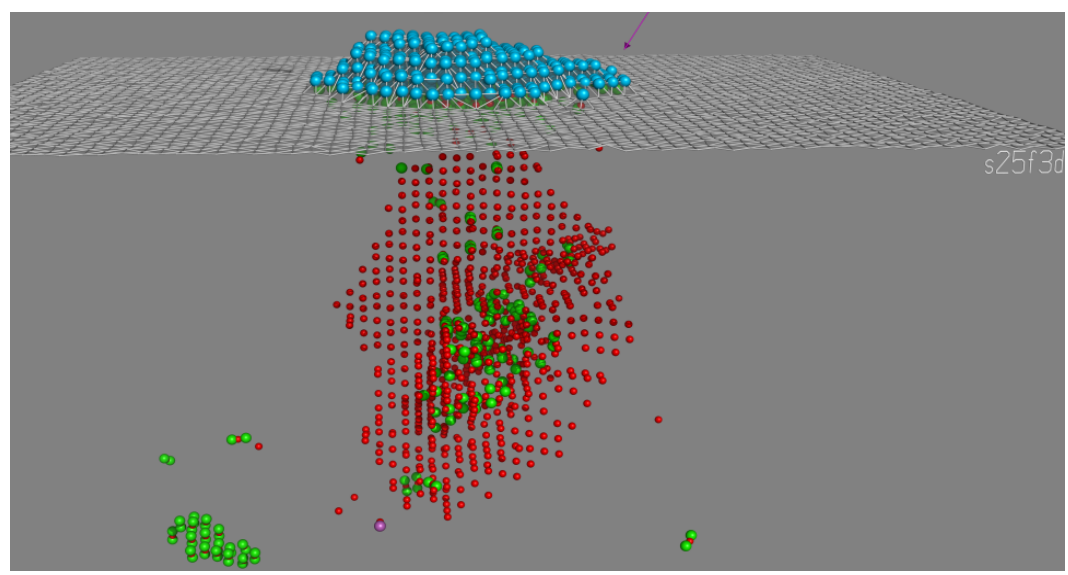

Fig.4b. 


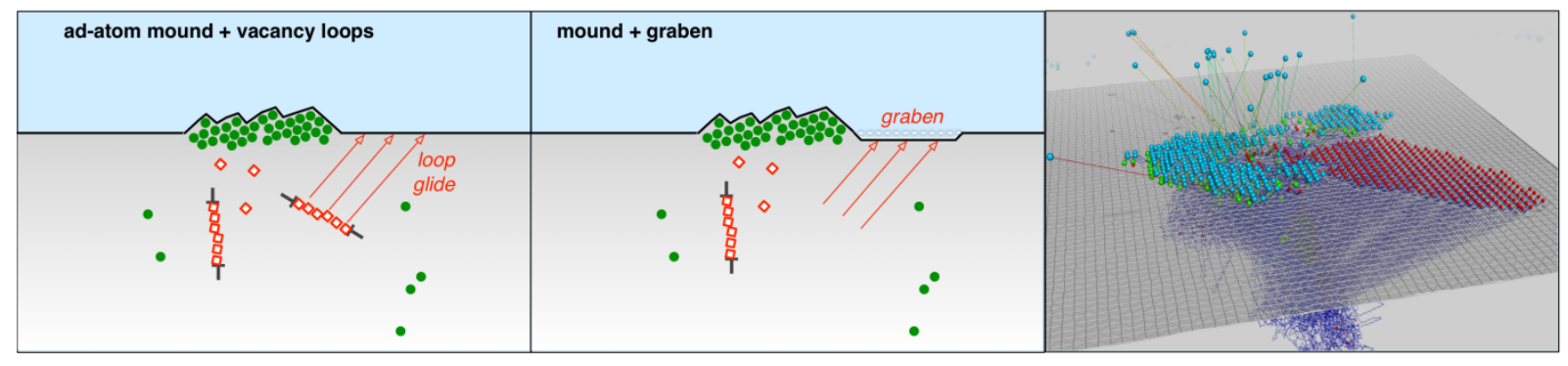

Fig.5a.

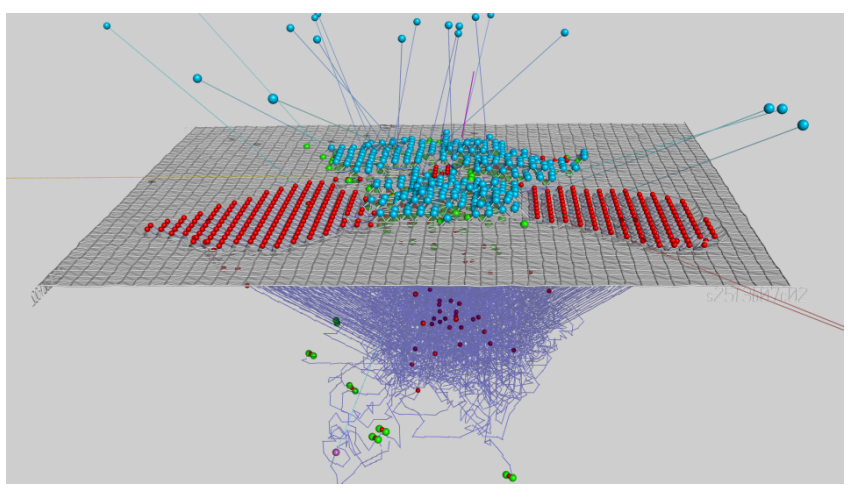

Fig.5b.

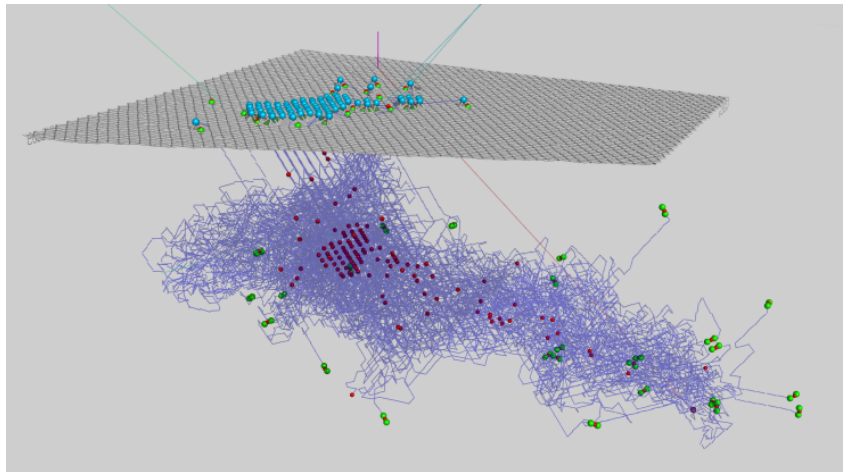

Fig.5c 


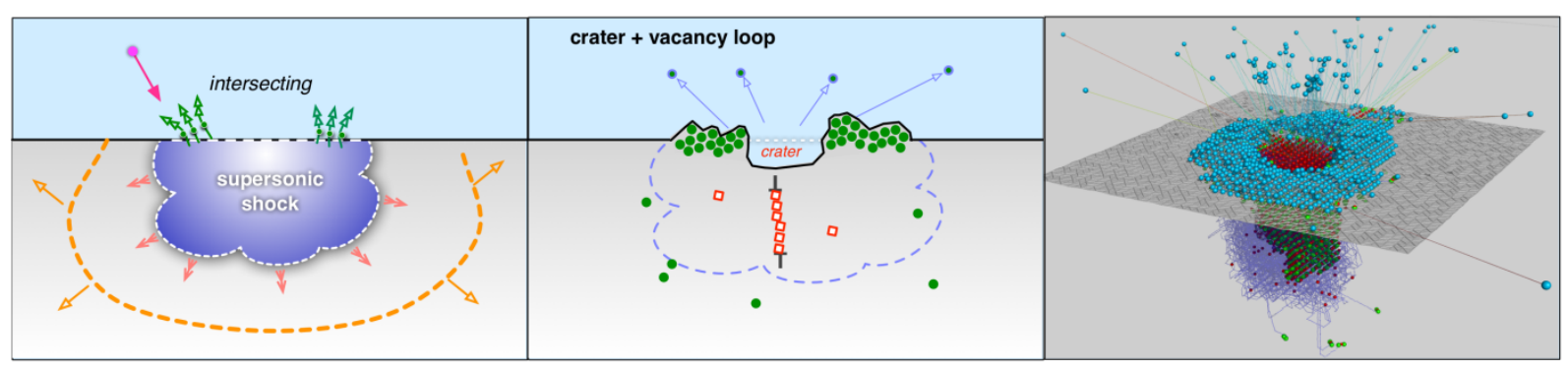

Fig.6a.

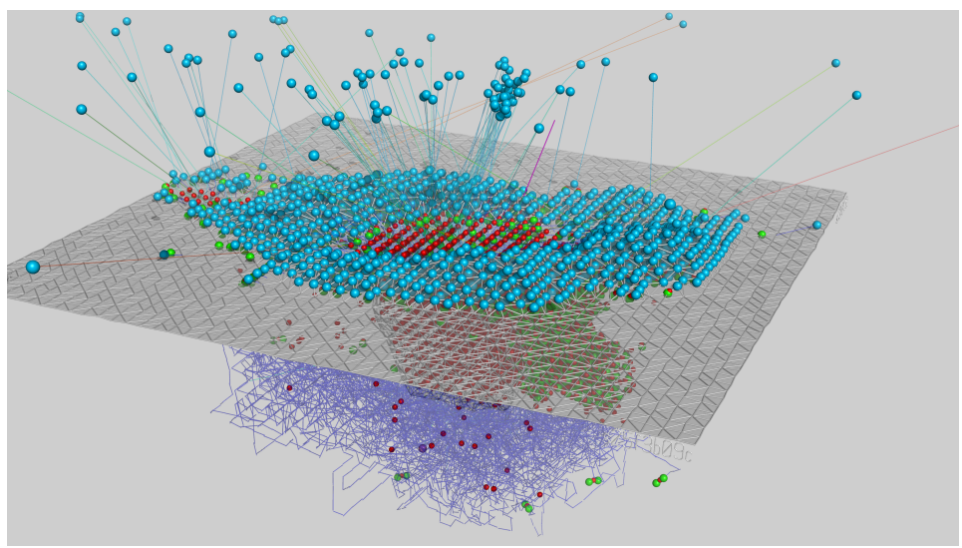

Fig.6b 


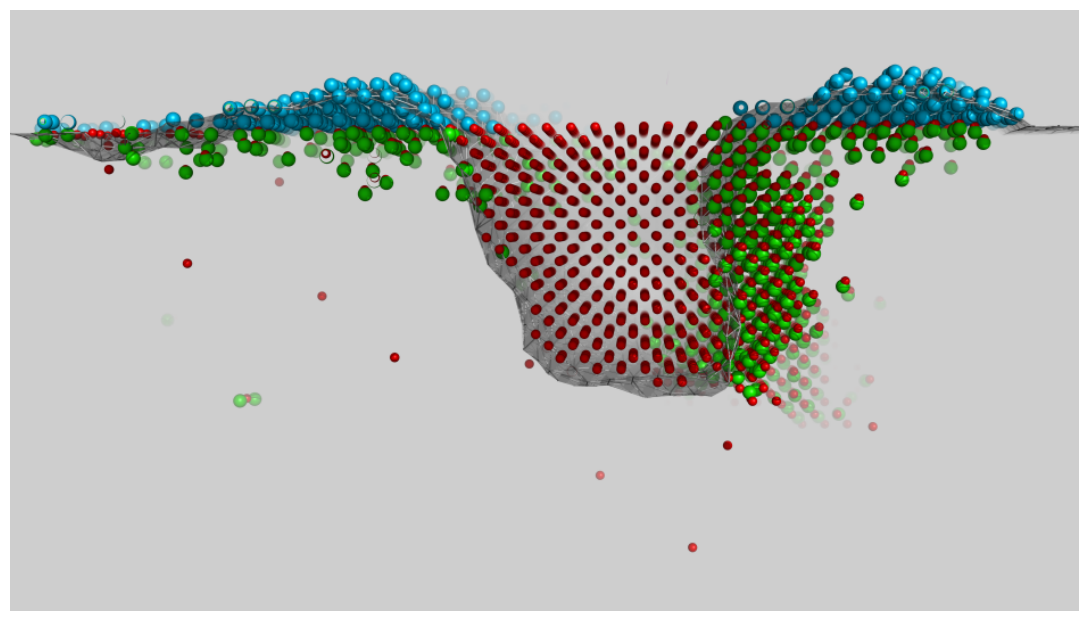

Fig.6c.

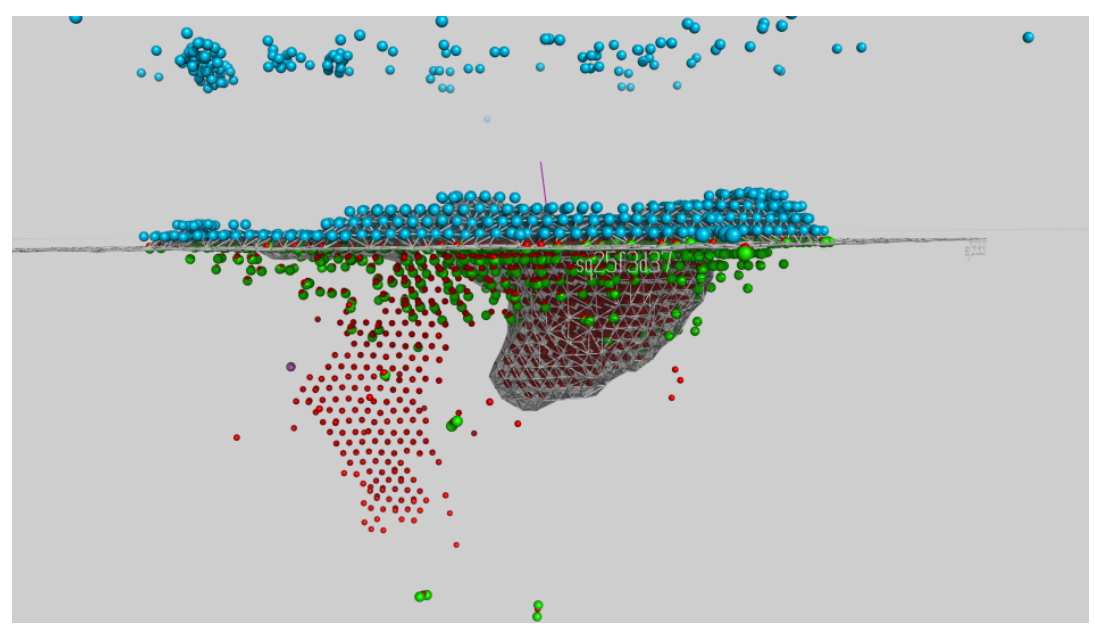

Fig.6d 


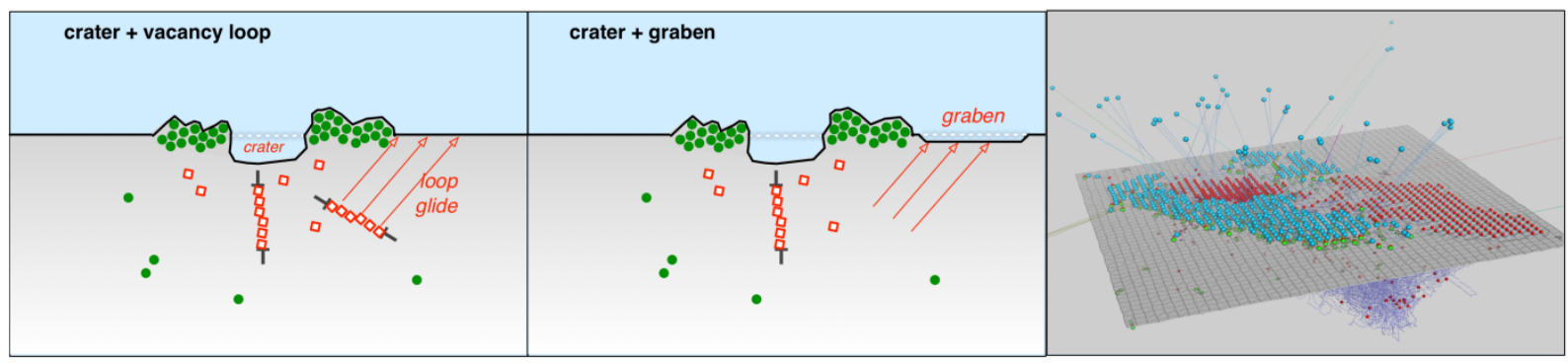

Fig.7a.

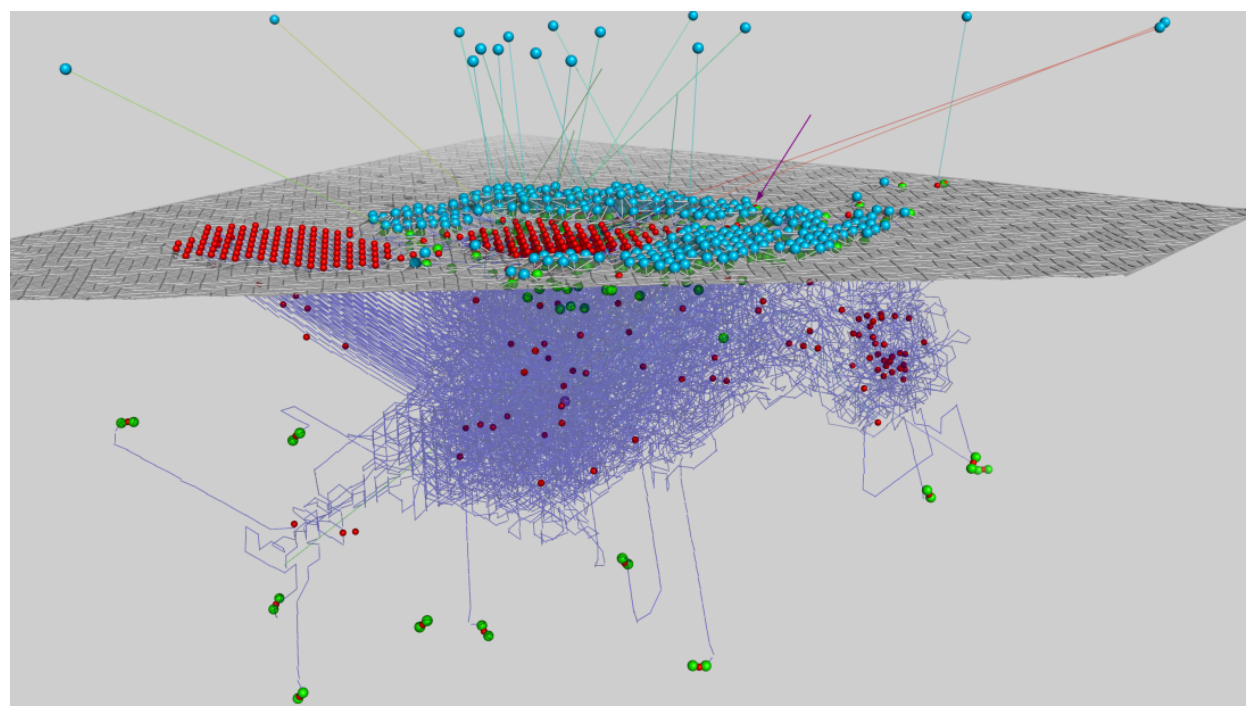

Fig.7b. 


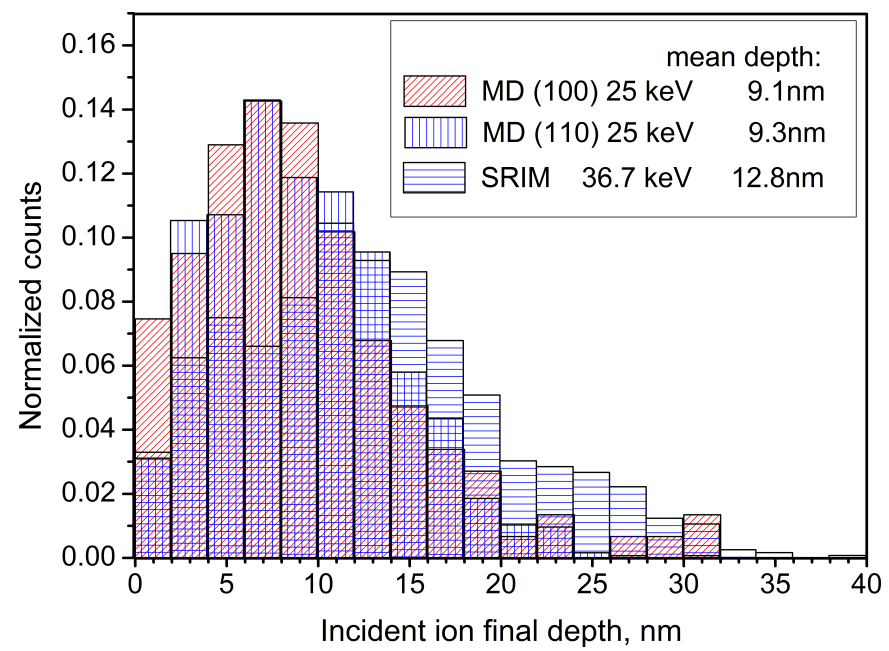

Fig.8. 